\title{
Acceleration of wound healing in gastric ulcers by local injection of neutralising antibody to transforming growth factor $\beta_{1}$
}

\author{
H Ernst, P Konturek, E G Hahn, T Brzozowski, S J Konturek
}

\begin{abstract}
Background-Application of neutralising antibodies (NAs) to transforming growth factor $\beta_{1}\left(\right.$ TGF $\left.\beta_{1}\right)$ improves wound healing in experimental glomerulonephritis and dermal incision wounds. TGF $\beta_{1}$ has been detected in the stomach, but despite the fact that this cytokine plays a central part in wound healing no information is available to determine if modulation of the TGF $\beta_{1}$ profile influences the healing of gastric ulcers. This study examines gastric ulcer healing in the rat after local injection of NAs to TGF $\beta_{1}$.

Method-Chronic gastric ulcers were induced in Wistar rats by the application of $100 \%$ acetic acid to the serosal surface of the stomach. Immediately after ulcer induction and on day 2 , NAs to TGF $\beta_{1}$ $(50 \mu \mathrm{g}), \mathrm{TGF} \beta_{1}$ (50 ng), saline or control antibodies (IgG; $50 \mu \mathrm{g}$ ) were locally injected into the subserosa. Controls received no subserosal injections. Animals were killed on day 5 or 11, the ulcer area was measured planimetrically, sections were embedded in paraffin wax, and stained with trichrome or haematoxylin and eosin. Depth of residual ulcer was assessed on day 11 by a scale of $0-3$, the percentage of connective tissue was determined by a semiquantitative matrix score and granulocytes and macrophages in the ulcer bed were also assessed.
\end{abstract}

Results-The application of NAs to TGF $\beta_{1}$ led to a significant acceleration of gastric ulcer healing on day $11(0.6$ (SD 0.8$) v 3.7$ (SD 2.6 ) $\mathrm{mm}^{2}$ ), a reduction in macrophages (23.7 (SD 22.6) $v 38$ (26) per $40 \times$ power field) and granulocytes (8.5 (SD 5.6) $v 20$ (10) per $40 \times$ power field), fewer histological residual ulcers (mean 1 (SD 0.9) $v 2$ $(1 \cdot 1))$, a reduced matrix score, and a regenerative healing pattern. Excessive scarring was seen in the TGF $\beta_{1}$ treated group.

Conclusion-Further treatment of gastric ulcers may induce a new treatment modality by local injection of NA to TGF $\beta_{1}$ in an attempt to accelerate and improve ulcer healing.

(Gut 1996; 39: 172-175)

Keywords: gastric ulcer, wound healing, $\mathrm{TGF} \beta_{1}$, neutralising antibodies to $T G F \beta_{1}$, quality of wound healing.

Fetal wounds heal with a reduced inflammatory and cytokine response and complete restitution of the normal tissue architecture. ${ }^{1}$ In contrast healing of adult wounds does not necessarily lead to complete restitution of normal structure and function. Studies in humans and animals have shown that pathological accumulation of extracellular matrix is a central biological feature of poor healing and poor preservation of the tissue architecture in interstitial lung fibrosis, glomerulosclerosis, scarring of skin wounds, and healing of gastric ulcers. ${ }^{2-7}$ Transforming growth factor $\beta_{1}$ $\left(\mathrm{TGF} \beta_{1}\right)$ has been shown to act as a molecular switch that turns the repair process on and off by exerting a variety of effects on the extracellular matrix. Data obtained from studies in animals and humans show that TGF $\beta_{1}$ plays a central part among cytokines in the stimulation of matrix production, inhibition of matrix degradation, and modulation of matrix receptors to increase cell adhesion to the matrix. ${ }^{38}$

In animal studies it has been shown that several growth factors applied to gastric ulcers, such as epidermal growth factor, $T G F \alpha$, platelet derived growth factor, and $b F G F$, accelerate the healing process by mechanisms that are not completely understood. ${ }^{9-12}$

TGF $\beta_{1}$ has been detected in the rat stomach $^{13}$ but no information is available at present, as to whether TGF $\beta_{1}$ has any influence on the healing of gastric ulcers. In experimental glomerulonephritis and dermal incision wounds, systemic application of neutralising antibodies (NAs) to TGF $\beta_{1}$ improved the healing process and reduced the deposition of extracellular matrix. ${ }^{36}$ Application of TGF $\beta_{1}$ to skin wounds in rats resulted in an increase in collagen synthesis within the wound, and accelerated rate of healing ${ }^{6} 14$ as with NAs. In this study we report that locally applied NAs to TGF $\beta_{1}$ in an experimental model of chronic gastric ulcer leads to an acceleration of ulcer healing, a reduction in extracellular matrix deposition, and an improvement in the restoration of tissue architecture.

\section{Methods}

Animal model

In all the experiments, chronic gastric ulcers were induced in male Wistar rats weighing $150-180 \mathrm{~g}$ by the method of acetic acid application to the serosa described elsewhere. ${ }^{11}$ Each group of animals contained 9-10 rats. The results were pooled for statistical analysis. \\ Medicine, University \\ Nuremberg, Erlangen, \\ H Ernst \\ Instite of Physiology, \\ School of Medicine, \\ Krakow, Pola \\ T Brzozowski
}


the first series 50 rats were divided into five groups and treated with local anti-transforming growth factor therapy or controls (as described below), were killed after day 5 , and the ulcer size was measured. In the second series 50 rats were divided also into the same groups and given the same treatments, but were killed on day 11. Tissues were removed and the ulcer size was measured and the ulcer area or scar was embedded in paraffin wax.

\section{Local anti-transforming growth factor therapy} Immediately after the induction of ulcers (during laparotomy) four of five groups, each containing 10 animals, received local (in the area of application of acetic acid) subserosal application of either $50 \mu \mathrm{g}$ NA to $T G F \beta_{1}$ (AB-101-NA, R and D Systems, Minneapolis, USA), 50 ng $\mathrm{TGF} \beta_{1}$ (BDP1, British Biotechnology, Oxford), $50 \mu \mathrm{g}$ chicken IgG-control (AB101-C, R and D Systems, Minneapolis, USA), or saline $(0.9 \%)$. The fifth group received no subserosal application.

On day 2 a laparotomy was performed and the NAs or control antibodies (and saline) were again injected locally into the ulcer area. These subserosal injections comprised the respective substance in phosphate buffered saline at a volume of $100 \mu \mathrm{l}$, and were applied just beside the ulcer on the same wall of the stomach. In one group, only a laparotomy was performed (sham operation).

\section{Specificity of $N A$ s to $T G F \beta_{1}$}

The specificity of the neutralising antibody against $\mathrm{TGF} \beta_{1}$ has been tested in direct ELISA and western blot analysis by $R$ and $D$ Systems. This antibody shows $10 \%$ cross reactivity with $\mathrm{TGF}_{5}$ and $<5 \%$ cross reactivity with $\mathrm{TGF} \beta_{3}$ and TGF $\beta_{2}$.

The concentration of neutralising antibody required to yield one half maximal inhibition of TGF $\beta_{1}$, when $T G F \beta_{1}$ is present to yield $100 \%$ activity, was tested in a neutralising bioassay using the murine T helper cell line, HT-2 cells. Three $\mu \mathrm{g} / \mathrm{ml}$ of antibody will neutralise $50 \%$ of the biological activity caused by $0.25 \mathrm{ng} / \mathrm{ml}$ of TGF $\beta_{1}$. Each batch of neutralising antibody to TGF $\beta_{1}$ has been tested in this bioassay by $R$ and $D$ Systems.

\section{Determination of ulcer size and depth}

The area of the ulceration was measured planimetrically by a person who was blinded to the origin of the coded specimens, using a computer planimeter (Morphomat 10, Opton, Germany), and results expressed in square millimetres. The sections were embedded in paraffin wax and stained with haematoxylin and eosin. In addition to measuring the surface area of the ulcer in millimetres, a scale of 0-3 was used to measure the depth of the residual ulcer $^{12}$ on day $11(0$, complete healing of the ulcer; 1, superficial erosion; 2, deep ulcer extending into the muscularis; and 3, penetrating or perforating ulcer).

\section{Morphometric analysis}

For the histological examination the ulcers were embedded in paraffin wax and stained with haematoxylin and eosin or trichrome.

\section{Matrix score}

On day 11 the area of dense connective tissue fibre material in the gastric ulcer bed in the trichrome stained sections was assessed by a semiquantitative score. This was expressed as the percentage of submucosal area covered by dense connective tissue fibre material. Two submucosal areas beneath the ulcer and two submucosal areas under normal mucosa were selected (100 point raster; with a $\times 100$ objective) by two independent observers. The results were pooled for statistical analysis. The results obtained in normal submucosa (same slides as healing ulcers) in the five different groups were used as control values.

\section{Morphometric analysis of macrophages and granulocytes}

On day 11 granulocytes were stained by leucocyte esterase, while macrophages were identified in the haematoxylin and eosin stain and were counted per low power $\times 40$ objective field in the ulcer bed.

\section{Statistical analysis}

For statistical analysis the non-parametric Mann-Whitney U and Kruskal-Wallis tests for unpaired comparisons were applied where appropriate.

\section{Results}

The mean ulcer size $\left(\mathrm{mm}^{2}\right)$ of animals treated with NAs to TGF $\beta_{1}$ was reduced slightly on days 5 (mean $7 \cdot 1$ (SD 3.2)) and greatly on day 11 (mean 0.6 (SD 0.84)) (Fig $1 \mathrm{~A}$ and $\mathrm{B}$ ). Surprisingly, treatment with $\mathrm{TGF} \beta_{1}$ alone delayed slightly ulcer size on day 5 , but also reduced the ulcer area significantly $(\mathrm{p}<0.05)$ on day $11(1.7(\mathrm{SD} \mathrm{1.6))} v$ controls (3.7 (SD $2 \cdot 6)$ ). Treatment with $\mathrm{TGF} \beta_{1}$, however, reduced the ulcer size to a smaller extent than treatment with NAs.

Eleven days after ulcer induction, histological assessment of residual ulcers by a score established by Szabo et al ${ }^{12}$ did show that the number of rats with histological residual ulcer was smaller only in the NA treated group (mean 1 (SD 0.9) $v$ controls 2 (SD 1.1)). The mean score of gastric ulcers in the sham control (mean $2 \cdot 1$ (SD $1 \cdot 0$ )), chicken IgG (mean 2 (SD 1.1)) or $\mathrm{NaCl}$ treated group (2.2 (SD 1.2)) showed almost no completely healed ulcers. In the group of animals treated with TGF $\beta_{1}$ (mean 1.7 (SD 1.0)) only two deep penetrating ulcers were seen. In the NA group (mean $1.0($ SD 0.9$)$ ), there were only three with residual ulcers and seven had completely healed ulcers or superficial erosions.

NA treated wounds had fewer macrophages (mean 23.7 (SD 22.6) v 38 (SD 26) in the controls) per low power field ( $\times 40$ objective) 
Figure 1: (A) Effect of treatment with NAs to $T G F \beta_{1}, T G F \beta_{1}$, or controls on the healing of chronic gastric ulcers on day 5 after induction of the ulceration ( $n=9-10$ each group). Results are expressed as means (SD). Error bars $=1 S D$. (B) Effect of treatment with $N A$ s to $T G F \beta_{1}, T G F \beta_{1}$, or controls on the healing of chronic gastric ulcers on day 11 after induction of the ulceration $(n=9-10$ each group). Results are expressed as means (SD). Asterisk shows significant $(p<0.05)$ decrease below the control value. Error bars $=1 S D$.
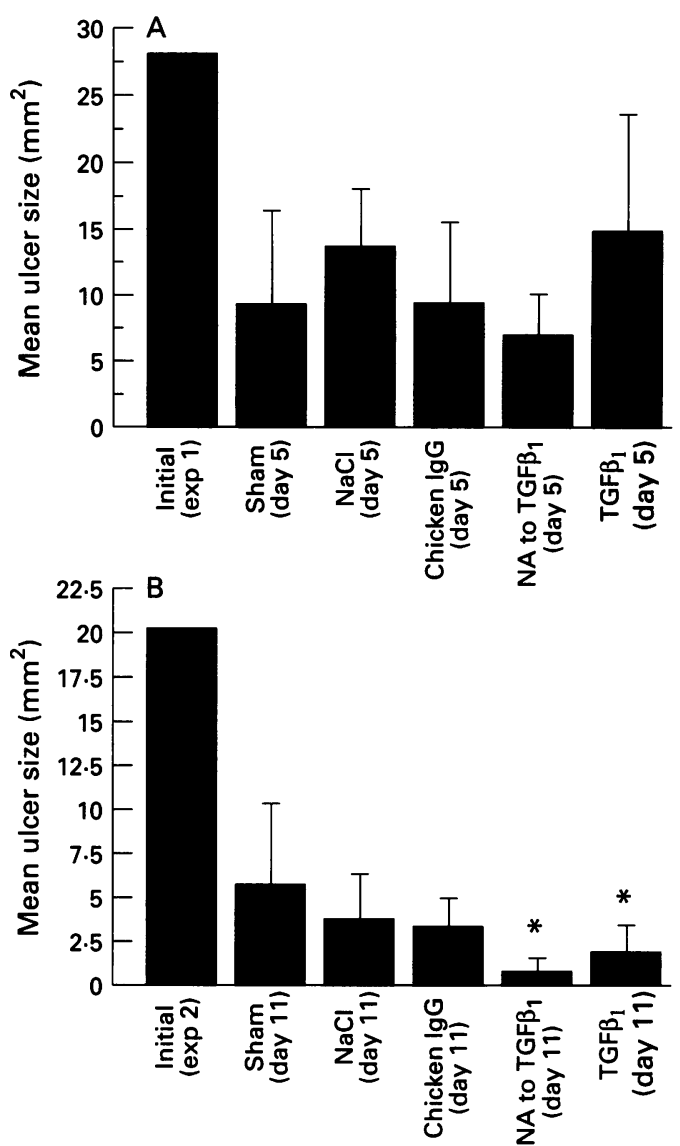

and fewer neutrophil granulocytes on day 11 (8.5 (SD 5.6) $v 20$ (SD 10)) per low power field ( $\times 40$ objective) than the controls. The number of macrophages and granulocytes in the ulcers treated with $\mathrm{TGF} \beta_{1}$ did not differ significantly from those in the controls.

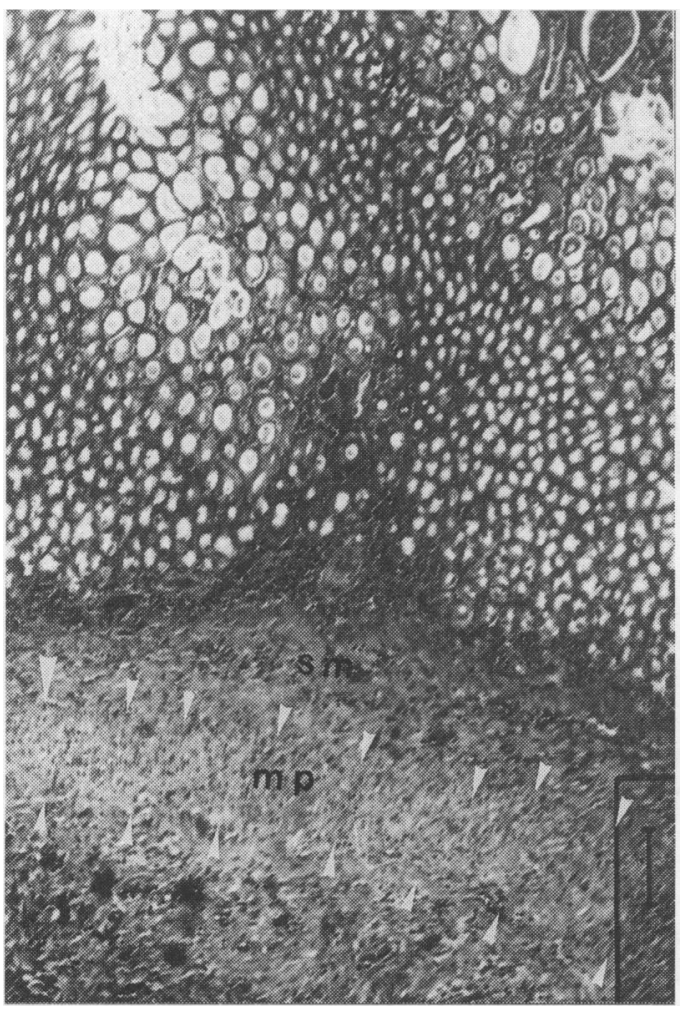

Figure 2: Gastric ulcer treated with NAs to TGF $\beta_{1}$. Regeneration of normal tissue architecture. Newly formed muscularis propria (arrows; $m p$ ) and small submucosa (sm) (original magnification $\times 25$ ). Bar $100 \mu \mathrm{m}$.

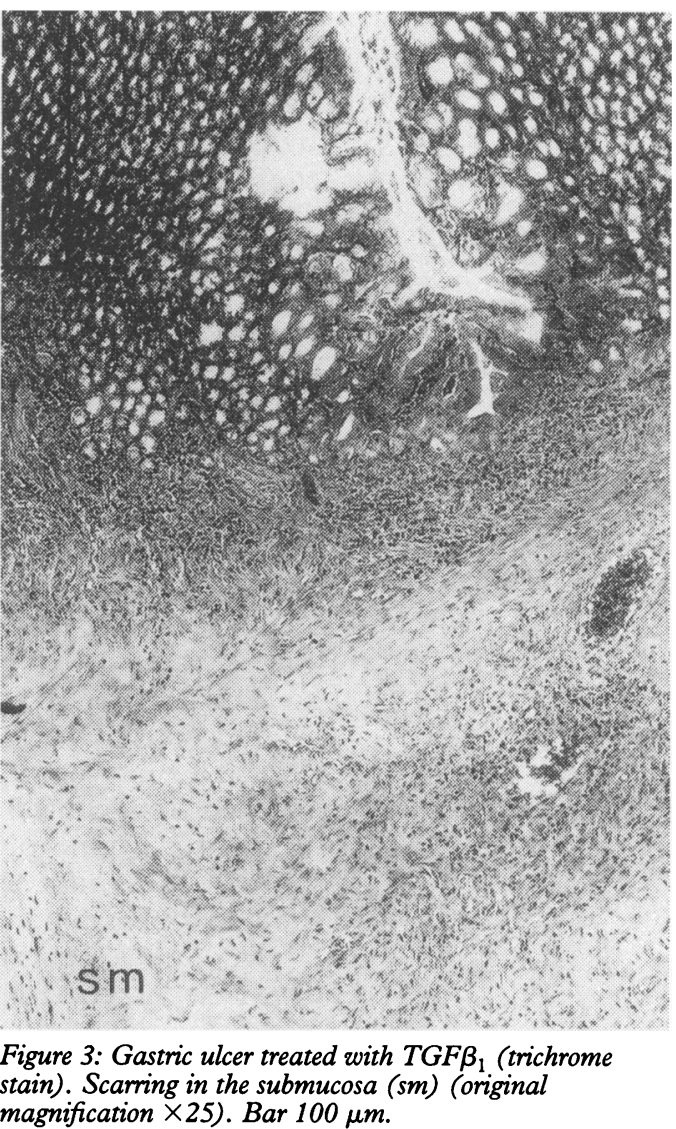

Histological examination (trichrome and haematoxylin and eosin stain) of the connective tissue compartment showed that ulcers treated with NAs had a more regenerative pattern of normal gastric mucosal architecture. Collagen fibre orientation in the gastric mucosa showed an almost normal regenerative pattern. A semiquantitative assessment of connective tissue fibres in the ulcer bed was made using a semiquantitative matrix score. The matrix score (mean of NA treated group $19 v$ 26 in the TGF $\beta_{1}$ treated group, and a mean of 23 in the control groups $v 13$ in normal submucosa) showed a less dense distribution of extracellular matrix in the submucosa of the ulcer bed in the NA treated group (Fig 2). The difference of the matrix scores between the NA to TGF $\beta_{1}$ treated group and the TGF $\beta_{1}$ treated group is statistically significant $(p<0.05)$. Apart from the matrix score the ulcers treated with $T G F \beta_{1}$ showed an orientation of collagen similar to that seen in scar tissue (Fig 3).

\section{Discussion}

Our experiments show that immediately after induction of gastric ulcers local application of $T G F \beta_{1}$ or NAs against $T G F \beta_{1}$ affects the rate of ulcer healing. Both lead to an acceleration of healing, but with different histological features - excessive scarring in $\mathrm{TGF} \beta_{1}$ treated ulcers versus a more regenerative healing pattern in NA treated ulcers.

The production and deposition of extracellular matrix is a key factor in tissue repair. ${ }^{5}$ TGF $\beta_{1}$ induces the formation of extracellular matrix components in a very complex way. It 
promotes extracellular matrix deposition not only by stimulation of extracellular matrix protein synthesis, but also by inhibition of protease synthesis, stimulation of protease inhibitor synthesis, and an increased synthesis of cell adhesion receptors, which function to bind a wide variety of extracellular matrix components. ${ }^{2}$

In gastroduodenal ulcer disease poor healing with submucosal scarring has been implicated as a factor of local ulcer recurrence. ${ }^{7}$ Shiann et al have shown that different therapeutic treatments may lead to different patterns of histological maturity. ${ }^{7}$ Tarnawski et al found that treatment with Maalox led to better submucosal healing and improved the overall quality of ulcer healing in contrast with treatment with $\mathrm{H}_{2}$ blockers. ${ }^{15}$

There is now evidence that overproduction of TGF $\beta_{1}$ may lead to scarring. ${ }^{561416}$ In evolutionary terms, it seems that adult wounds may be optimised for speed of healing and this may result in an excessive $T G F \beta_{1}$ release. In response to tissue damage $T G F \beta_{1}$ is released by platelets. TGF $\beta_{1}$ induces local cells to produce extracellular matrix and more $T G F \beta_{1}$. A potential regenerative response may be overrun by a cytokine surplus leading to scar formation. 258

Evidence of a failure in the regulation of TGF $\beta_{1}$ has been implicated in experimental glomerulonephritis and disorders associated with interstitial fibrosis. ${ }^{35}$ Systemic application of $T G F \beta_{1} N A s$ prevented autoinduction of TGF $\beta_{1}$-mRNA, and limited the inflammatory response in experimental glomerulonephritis, and decreased the deposition of extracellular matrix. ${ }^{3}$ In contrast with adult wounds with an excess of cytokines, cytokine profiles in fetal wounds are reduced. ${ }^{1}$ Here we show that lowering the growth factor concentrations of $\mathrm{TGF} \beta_{1}$ in a chronic gastric ulcer model leads to faster healing with considerably diminished scarring and infiltration of the ulcer base with fewer macrophages and granulocytes.

In contrast, local application of $\mathrm{TGF} \beta_{1}$ resulted in massive scar formation. Recently, Mustoe et al showed that treatment of partial thickness gastric serosal incisions with a single dose of TGF $\beta_{1}$ accelerated the healing of the wounds. ${ }^{16}$ In our experiments, application of $\mathrm{TGF} \beta_{1}$ accelerated the overall healing rate compared with the control groups.

This finding may be explained by the hypothesis that during the process of evolution adult wounds may have been optimised for speed of healing under adverse conditions. This may have resulted in excessive inflammatory infiltrates and an excess of cytokines during wound healing in the adults. ${ }^{2}$ Shah et al mimicked this fetal wound situation in the healing adult rat skin wound by local injection of an anti-TGF $\beta_{1}$ NA to reduce TGF $\beta_{1}$ concentrations. This resulted in diminished scarring in adult wounds and almost normal dermal architecture. Injection of TGF $\beta_{1}$ alone had the opposite effect. ${ }^{6}$

Macrophages and activated platelets are the principal source of $T G F \beta_{1}$ in the adult wound.
In the foetus, wounds heal with very little inflammation, and the reduced concentration of TGF $\beta_{1}$ may reflect the absence or minimal macrophage infiltrates in those wounds. ${ }^{25}$

Our study showed a reduction in the number of macrophages in wounds treated with NAs. But in wounds treated with TGF $\beta_{1}$ there was no significant difference in the number of macrophages compared with controls.

For ulcer relapse, excessive deposition of scarring tissue has been implicated as a risk factor. ${ }^{4}$ Here we show that lowering the active growth factor concentration of $\mathrm{TGF} \beta_{1}$ by local application of NA at the ulcer site accelerates ulcer healing and improves the arrangement of the tissue architecture of the healing ulcer.

Our findings suggest that an anti-TGF $\beta_{1}$ therapy modality in gastric ulcer disease may be a novel means of stimulating and improving ulcer healing.

A preliminary report on this work was presented in abstract form at the Digestive Disease Week, San Diego, 14-17 May 1995 .

This work was supported by a grant from the $\mathrm{J}$ and $\mathrm{F}$ This work was
Marohn-Stiftung.

1 Whitby DJ, Ferguson MWJ. Immunohistochemical localisation of growth factors in fetal wound healing. Dev Biol 1991; 147: 207-15.

2 Adzick NS, Lorenz HP. Cells, matrix, growth factors, and the surgeon. The biology of scarless fetal wound repair. Ann Surg 1994; 220: 10-8.

3 Border WA, Okuda S, Languino LR, Sporn MB, Ruoslahti E. Suppression of experimental glomerulonephritis by antiserum against transforming growth factor $\beta 1$. Nature 1990; 346: 371-4.

4 Ishimori A, Kawakami K, Inoue S, Kano A, Takahashi T, Asaki S, et al. Predictors of relapse in peptic ulcer. Hepatogastroenterology 1992; 39: 396-9.

5 Roberts AB, Sporn MB. The transforming growth factorBs. In: Sporn MB, Roberts AB, eds. Peptide growth factors and their receptors I. Berlin: Springer Verlag, 1990: 421-32.

6 Shah M, Foreman DM, Ferguson MWJ. Control of scarring in adult wounds by neutralising antibody to transforming growth factor $\beta$. Lancet 1992; 339: 213-4.

7 Shiann P, Liao CH, Chen SH. Histological maturity of healed duodenal ulcers and ulcer recurrence after treathealed duodenal ulcers and ulcer recurrence after treat-
ment with colloidal bismuth subcitrate or cimetidine. ment with colloidal bismuth subcit
Gastroenterology 1991; 101: 1187-91.

8 Border WA, Brees D, Noble NA. Transforming growth factor-beta and extracellular matrix deposition in the kidney. In: Koide $\mathrm{H}$, Hayashi $\mathrm{T}$, eds. Extracellular matrix in the kidney. Basel: Karger, 1994: 140-5.

9 Guglietta A, Hervada T, Nardi C, Lesch A. Effect of platelet-derived growth factor-BB on indomethacininduced gastric lesions in rats. Scand $\mathcal{F}$ Gastroenterol 1992; 27: $673-6$.

10 Konturek SJ, Brzozowski T, Majka J, Dembinski A, Slomiany A, Slomiany BL. Transforming growth factor alpha and epidermal growth factor in protection and healing of gastric mucosal injury. Scand $\mathfrak{f}$ Gastroenterol healing of gastric

11 Konturek SJ, Dembinski A, Warzecha Z, Brzozowski T, Gregory $\mathrm{H}$. Role of epidermal growth factor in healing of chronic gastroduodenal ulcers in rats. Gastroenterology 1988; 94: 1300-7.

12 Szabo S, Folkman J, Vattay P, Morales RE, Pinkus GS, Kato K. Accelerated healing of duodenal ulcers by oral administration of a mutein of basic fibroblast growth factor in rats. Gastroenterology 1994; 106: growth 11 .

13 Kobayashi K, Tominaga K, Kim S, Fukuda T, Higuchi K, Nakamura $\mathrm{H}$, et al. Expression of genes for transforming growth factor- $\beta 1$ and components of the extracellular matrix during gastric ulcer healing in rats. Gastroenterology 1994; 106: 108A.

14 Mustoe TA, Pierce GF, Thomson A, Gramates P, Sporn MB, Duel TF. Accelerated healing of incisional wound induced by transforming growth factor- $\beta$. Science 1987 237: 1333-6.

15 Tarnawski A, Stachura J, Krause WJ, Douglass TG, Gergely $\mathrm{H}$. Quality of gastric ulcer healing: a new, emerg ing concept. $\mathcal{F}$ Clin Gastroenterol 1991; 13 (suppl 1): S42-7.

16 Mustoe TA, Landes A, Cromack DT. Differential acceleration of healing of surgical incisions in the rabbit gastrointestinal tract by platelet-derived growth factor and transforming growth factor, type $\beta$. Surgery 1990; 108: 324-30. 